\title{
Technical feasibility study for a solar energy system at Amsterdam Airport Schiphol (AAS)
}

\author{
P. Janssen ${ }^{1}$, J. Myrzik ${ }^{2}$,W. Kling ${ }^{3}$, L. Reinders ${ }^{4}$ \\ ${ }^{1,2,3}$ Department of Electrical Engineering \\ Technical University Eindhoven \\ PO Box 513, CR. 2.04, 5600 MB Eindhoven, the Netherlands
}

Phonenumber:+0031 206019140 , e-mail: janssen_p@schiphol.nl,W.L.Kling@tue.nl, johanna.myrzik@tu-dortmund.de

\author{
${ }^{1,4}$ Schiphol Group \\ Amsterdam Airport Schiphol \\ PO Box 7501, 1118 ZG Schiphol, the Netherlands \\ Phonenumber:+0031 206019140 , e-mail: janssen_p@ schiphol.nl, reinders_1@ schiphol.nl
}

\begin{abstract}
.
This work arises within the framework of the introduction of renewable energies at Amsterdam Airport Schiphol (AAS) and the focus is on the technical feasibility of a solar energy system (SES) on ground level. The flight safety must always be guaranteed if solar panels are implemented on AAS. The radiation reflection of solar panels according to the optical effect towards the pilot of an airplane and the line of sight of the Air traffic tower has been studied. Also the constraints for installing an "object" at the Airport have to be taken into account. This should lead to an overall impression of the potential of solar panels that can be orientated on ground level. A complete model of a solar energy system is designed: tilt angle, type of solar panels, equipment (DC/AC inverter), a cable system as well as the way the design can be implemented in the electricity infrastructure of AAS. Power Quality (PQ) and the effects of implementing a SES will be discussed: disturbances have to be minimized.
\end{abstract}

\section{Key words}

Constraints, reflection, radiation, solar energy system and power quality.

\section{Introduction}

THIS document describes a technical feasibility study for solar panels that can be implemented at ground level at Amsterdam Airport Schiphol (AAS). Schiphol Group, owner of AAS, has set up a climate plan [1] regarding decreasing $\mathrm{CO}_{2}$ and $\mathrm{NO}_{\mathrm{X}}$ emissions with a target in terms of amount of renewable energy that needs to be implemented at the Airport itself up to 2020. It is very important for Schiphol Group to be aware of the climate problems: AAS wants to be the most preferred Airport in the world also regarding the climate issues. If this would not be taken into account, it will have a negative effect on the increase of the flight movements. Two specific plans have also been setup: a Blue Energy Plan and a Mobility Plan. In these plans, a description is made for a path that should lead to the ambitions settled down in the Climate plan for 2020. This research is part of the Blue Energy plan because the latter one describes the feasibility of renewable energy and energy efficiency. Schiphol Group wants to generate at least $20 \%$ of its energy demand in 2020 by renewable energy sources. This renewable energy target can be reached by implanting solar panels, high efficient thermal energy installation (CHP), geothermic energy and also wind turbines. However wind energy will not be part of the $20 \%$ renewable target because it can not be installed at the Airport itself. Besides this, efforts for energy savings are and will be taken otherwise the amount of renewable energy that needs to be installed would be much higher. At the airport, it is not possible to implement all kinds of renewable innovations because of the flight safety regulations which define some constraints. These constraints have to be taken into account by the decision whether or not solar panels can be implemented on ground level. In this paper the maximum usable surface on ground will be investigated. For one small area a possible implementation of a grid-connected solar installation will be discussed. Furthermore the effect of the optical reflections of a solar panel will be part of the research as well as some aspects concerning Power Quality problems and grounding.

\section{Constraints}

For implementing objects like solar panels on ground level at AAS, constraints need to be taken into account. These constraints or guidelines are edited and owned by the International Civil Aviation Organization (ICAO) [2] and for the Netherlands, by the Dutch Air traffic control (LVNL) [3]. The guidelines for communication, radarand surveillance equipment (CNS) and obstacle freezones are explained in the ANNEX 10/14, EURODOC015 and the VOBA version 1.0. The most 
important communication system, the Information Landing System (ILS), is used to make a precise and safe landing at the runway. The landing can also be done completely automated by the usage of the ILS system and even in bad weather conditions. The ILS system involves three different communication systems, see figure 1:

- Localizer (LOC)

- Glide path antenna (GP)

- Precision approach path indicator (PAPI)

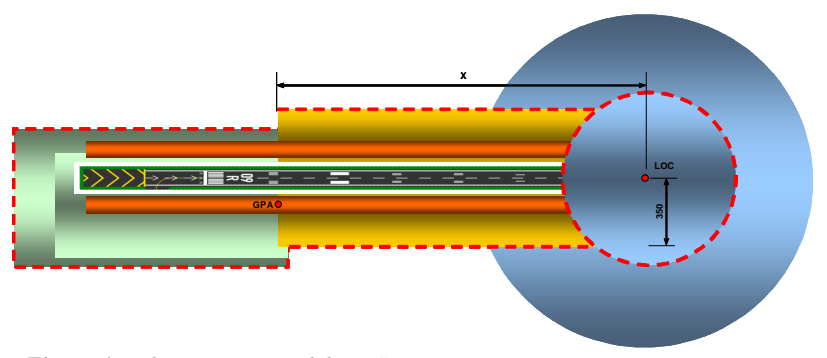

Figure 1 The constraints of the ILS system

A GP transmits the correct coordinates, dual frequency, to the airplane with the information of the angle of approach towards runway. In this way the pilot knows if the position of the airplane is correct according to the angle of approach. A LOC is an omni directional antenna and positioned symmetrically at the end of the runway. There are more antennas positioned at the end of the runway. The LOC transmits two frequency signals and depending on the signal strength at the receiver in the airplane, the pilot knows if the airplane will be in the middle of the runway or not. All Communication, Radar and Surveillance systems (CNS) have been analyzed and the surface availability for implementing solar panels at ground are presented in figure 2 .
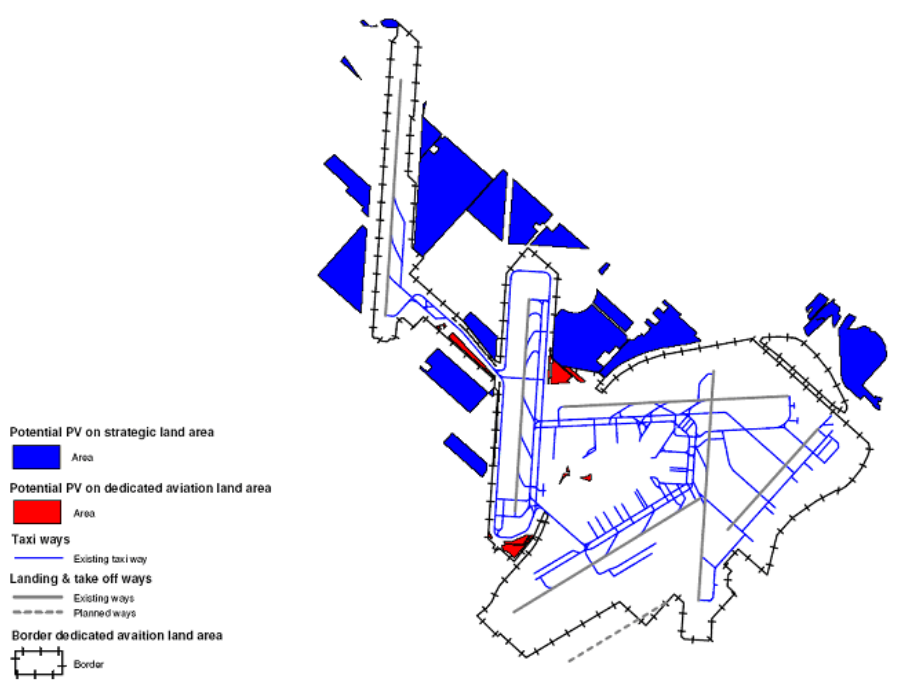

Figure 2 The amount of availability that can be used for solar panels

The total available surface can be divided into two areas:

- Blue $\rightarrow 605$ hectares, ground that is not belonging to the aerodrome but owned by Schiphol Group, so called strategy land area.

- $\quad$ Red $\rightarrow 25$ hectares for dedicated aviation land area according the LVNL constraints. For the
- ICAO constraints it would be 125 hectares but at AAS this is not the case because of the LVNL guidelines.

The total surface that can be used for implementing solar panels regarding the constraints of LVNL and ICAO on ground level is therefore 630 hectares. The ICAO constraints are minimum guidelines and they will always be overruled by the Dutch Air traffic control guidelines.

\section{Reflections}

For the flight safety it is necessary to know how much the optical impact will be according to the reflection of the sun by the use of solar panels. Reflection can lead to irritation or blindness of the human eye. For the flight safety it is important that the amount of optical reflection is minimized. The solar panel has an anti-reflection layer that minimizes the amount of irradiation reflection of the direct solar beam. The amount is not only depending on the reflection layer but also on the index of refraction of the material. For a perpendicular directional radiation beam, reflections on the glass surface will appear. The amount of reflection depends on the layer itself and this gives a reduction of the reflection. For the human eye the effect of the reflection only depends on the distance of the observer in relation to the solar panel. The human eye is the most sensitive for a wavelength of $550 \mathrm{~nm}$, the color green [4]. There is a relation between the solar radiation and the illuminance of a light source. In figure 3 , the human eye is represented in relation to the light source.

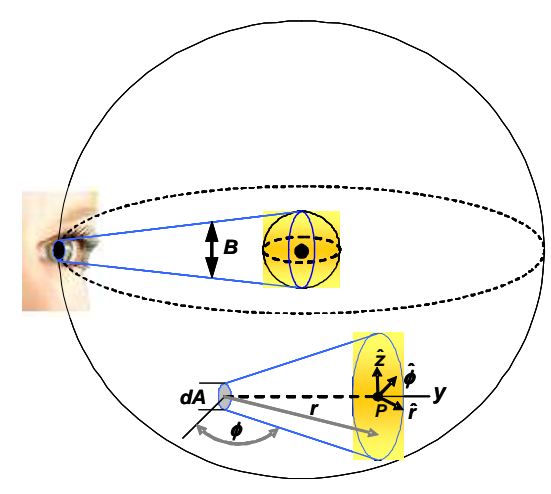

Figure 3 The human eye related to a light source

The impact of single glass versus antireflection glass on the illuminance is presented in figure 4 . This illuminance depends on the distance according to the human eye. It can be concluded that an anti-reflection layer decreases the amount of reflection. 


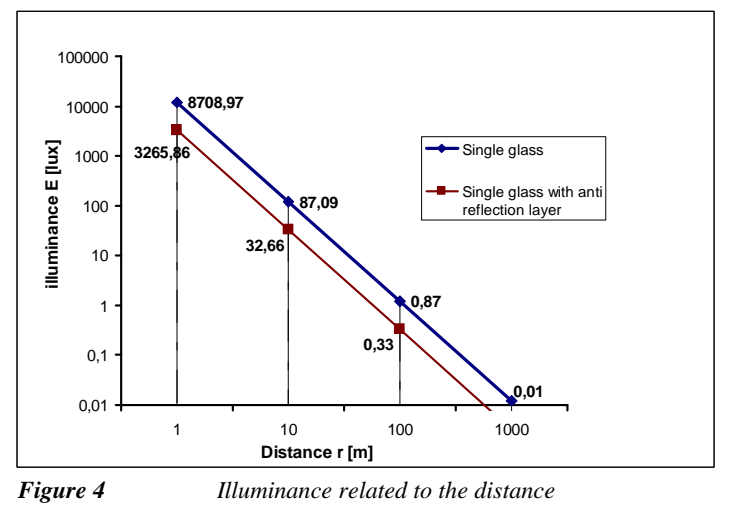

The reflection of the radiation at the solar panel is relatively small and this means that the effect normally can be neglected for the flight safety. The reflection coefficient of the solar panel is chosen to be $\leq 3 \%$ to minimize the effect.

\section{Radiation}

The amount of solar radiation depends upon the distance between the sun and the earth. The visibility of light for the human eye varies between 400 and 800 nanometers. To calculate the amount of radiation which can be used for solar panels, the global radiation is used. The global radiation $E$ is the sum of the direct- and diffuse radiation and is measured on a horizontal surface. The knowledge of the sun's path and solar azimuth according to the angle of inclination is important for calculating the optimal tilt angle and orientation angle of the solar panel. The tilt angle $\gamma_{P}$ and the orientation angle $\alpha_{P}$ can be calculated according to (1). The different angles are defined in figure 5 [5].

The optimal tilt $\gamma_{P}$ depends on the global radiation $E=E_{\perp} \cos \theta$, where $E_{\perp}$ is the global radiation measured on a horizontal surface.

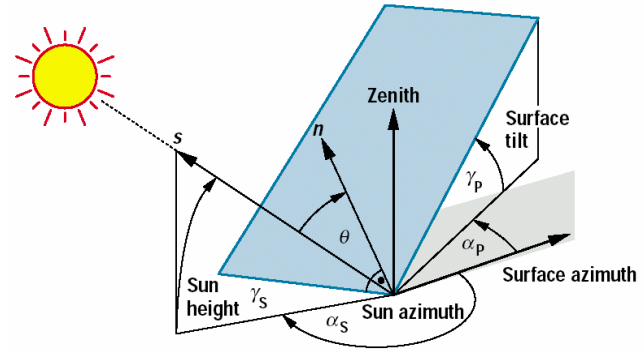

Figure 5 Different angles for an inclined surface

$$
\begin{aligned}
& s=\left(\cos \alpha_{S} \cdot \cos \gamma_{S},-\sin \alpha_{S} \cdot \sin \gamma_{S}, \sin \gamma_{S}\right)^{T} \\
& n=\left(-\cos \alpha_{P} \cdot \sin \gamma_{P}, \sin \alpha_{P} \cdot \sin \gamma_{P}, \cos \gamma_{P}\right)^{T} \\
& \cos \theta=s \bullet n \\
& \cos \theta=-\cos \alpha_{S} \cdot \cos \gamma_{S} \cdot \cos \alpha_{P} \cdot \sin \gamma_{P}- \\
& \sin \alpha_{S} \cdot \cos \gamma_{S} \cdot \sin \alpha_{P} \cdot \sin \gamma_{P}+\sin \gamma_{S} \cdot \cos \gamma_{P}
\end{aligned}
$$

The global radiation $E$ will be the highest when $\cos \theta$ is maximal. The calculation of the tilt angle depends on:
- The orientation angle which varies from $0^{0}<\alpha_{P} \leq 360^{\circ}$, is comparable with the sun azimuth.

- The day of the year and the time of the day. The radiation energy on a horizontal surface is the best for the time interval 10:00<t $\leq 14: 00$, see figure 6 .

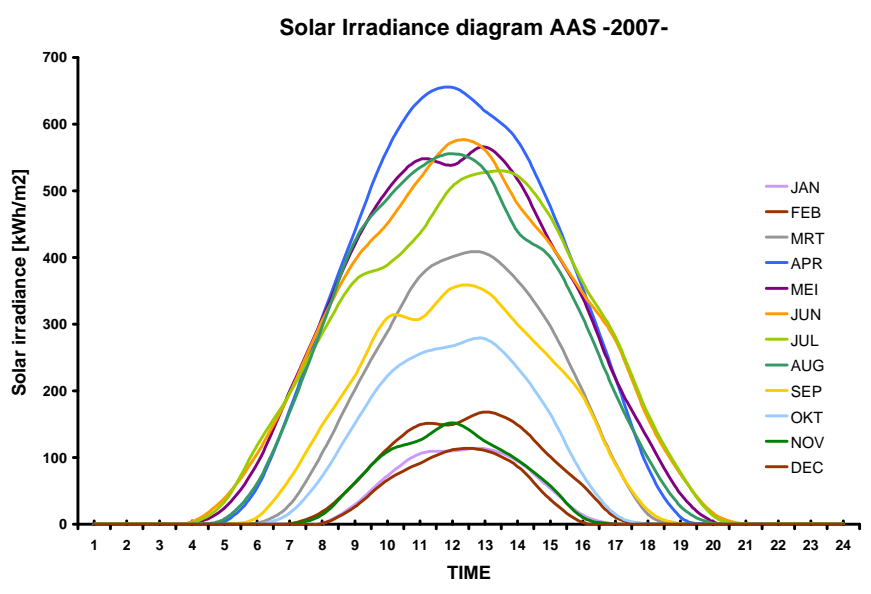

Figure 6 Measured solar irradiance $E_{\perp}$ on a horizontal surface at AAS

From the given equation, the 4 variables are correlated according to:

- $\cos \theta=f\left(\gamma_{s}, \alpha_{s}, \gamma_{P}, \alpha_{P}\right)$

Nevertheless, $\gamma_{S}$ and $\alpha_{S}$ are fixed; $\gamma_{P}$ and $\alpha_{P}$ are therefore the only two variables

- $\cos \theta=f\left(\gamma_{P}, \alpha_{P}\right)$.

The maximum of this equation needs to be calculated. Therefore the Hessian matrix (2) has to be drawn up.

$$
\begin{aligned}
& H=\left[\begin{array}{cc}
\frac{\partial^{2} f}{\partial \alpha_{P}^{2}} & \frac{\partial^{2} f}{\partial \alpha_{p} \partial \gamma_{P}} \\
\frac{\partial^{2} f}{\partial \gamma_{P} \partial \alpha_{p}} & \frac{\partial^{2} f}{\partial \gamma_{P}^{2}}
\end{array}\right] \Rightarrow \\
& r=\frac{\partial^{2} f}{\partial \alpha_{P}^{2}} \quad, \quad s=\frac{\partial^{2} f}{\partial \alpha_{p} \partial \gamma_{P}} \quad, \quad t=\frac{\partial^{2} f}{\partial \gamma_{P}^{2}}
\end{aligned}
$$

(a) $\frac{\partial f}{\partial \gamma_{P}}=0 \wedge \frac{\partial f}{\partial \alpha_{P}}=0$

(b) Maximum:

$$
\operatorname{det}(H)>0 \wedge s^{2}-r \cdot t<0 \wedge r<0
$$

(c) Minimum:

$$
\operatorname{det}(H)>0 \wedge s^{2}-r \cdot t<0 \wedge r>0
$$

(d) Saddle point: $\operatorname{det}(H)<0 \wedge s^{2}-r \cdot t>0$

The function admits a maximum if and only if the conditions (a) and (b) are valid. For the orientation angle $\alpha_{P}$, it seems that it only depends on the sun azimuth $\alpha_{S}$, therefore: $\alpha_{P}=180^{\circ}+\alpha_{S}$. The optimum is not only valid for a mean value but it also depends on the amount of sun hours on a specific day. Otherwise, according to the amount of sun hours, it would have a negative effect on the yearly yield of the solar energy system. According to the equation $E=E_{\perp} \cos \theta$, the maximum is obtained 
for $\cos \theta=1$. The maximum can be calculated for different tilt- and orientation angles for a fixed $E_{\perp}=1000 \mathrm{~W} / \mathrm{m}^{2}$. It seems that maximum radiation intensity $E$ is reached for a tilt $\gamma_{P}=31^{0}$ and $\alpha_{P}=180^{\circ}$ (south-orientation).

\section{Solar energy system at AAS}

A solar energy system (SES) model that can be implemented in the infrastructure of AAS will be developed. This model is designed by the use of a process model, see figure 7. A SES can have two options: a grid connected- or standalone system. Regarding the availability of the electrical infrastructure at AAS, a grid connected system has been chosen.

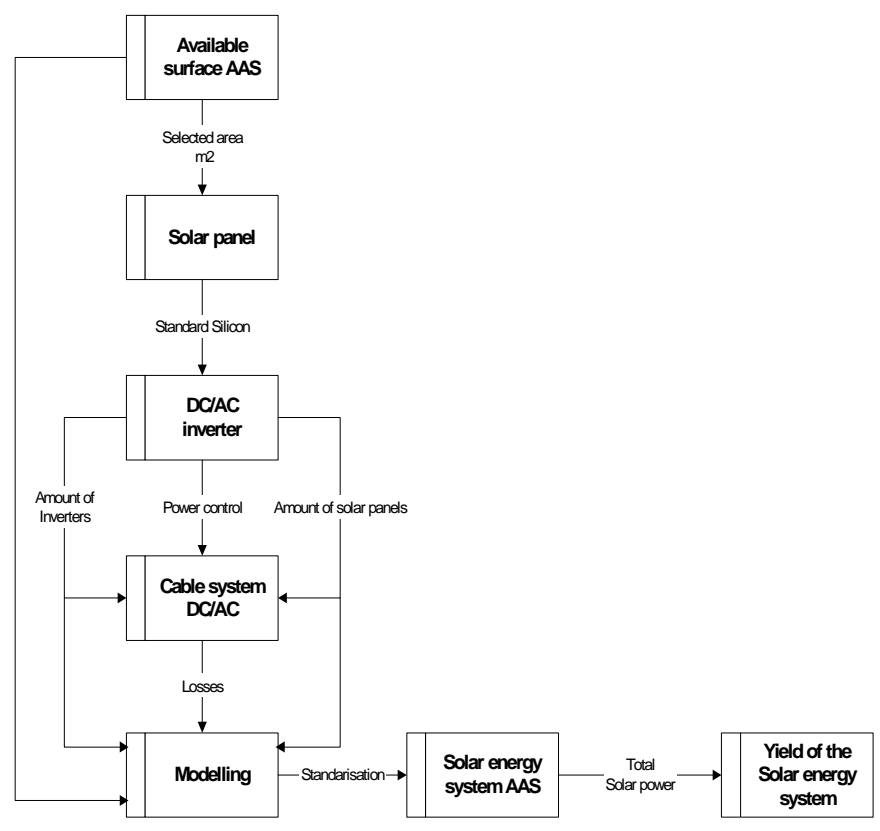

Figure 7 Process model to develop the solar energy system model

A typical solar system, see figure 8 could be used in the electrical infrastructure of AAS [6]. The Solar model is a three phase symmetrical system, where every inverter is connected as one phase. Hereby it is important that the total inverter system can be divided by 9 because of a three phase symmetrical system. The location of implementation, a grid connected solar system, could be the area North - West, see figure 9. This area is divided into 4 sub areas and every sub area can have an amount of solar panels. The numbers of solar panels on a solar construction per sub area are given in table 1 .

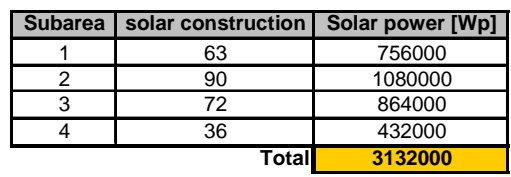

Table 1 Total solar power

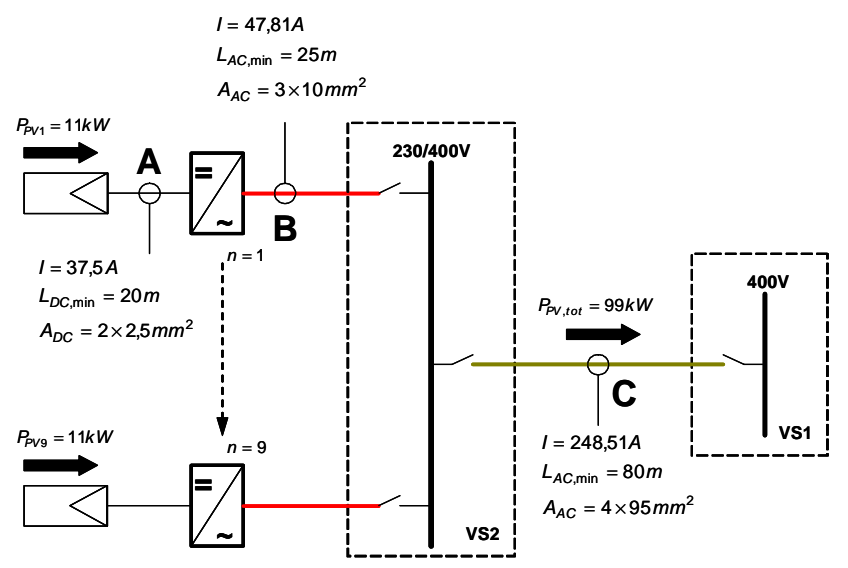

Figure $8 \quad$ Layout solar system

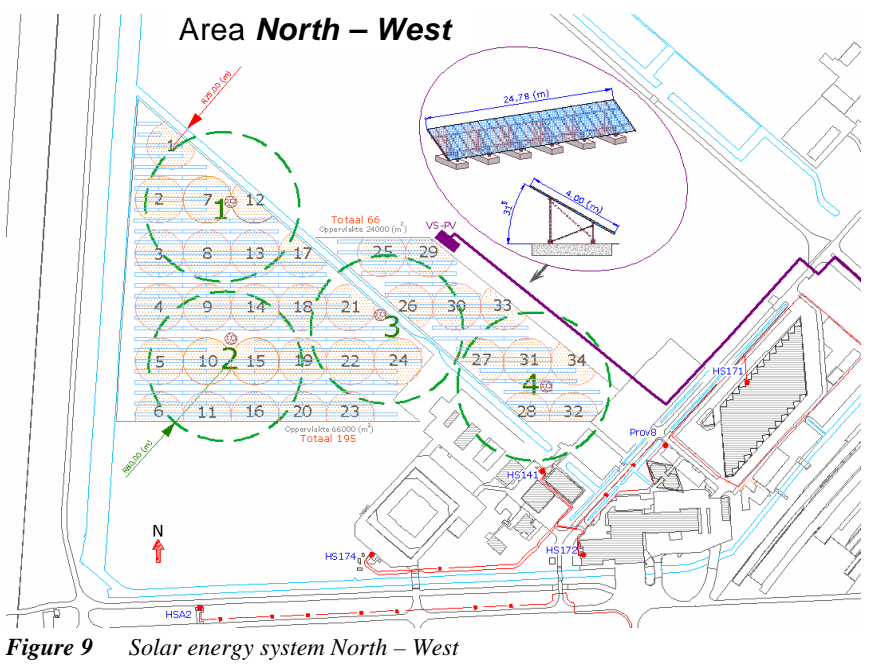

The total solar power that can be installed in the North West area is $3,132 \mathrm{MWp}$ (solar panel of $200 \mathrm{Wp}$ ). In every sub area a $10 \mathrm{kV} / 400 \mathrm{~V}$ distribution transformer must be installed. The yearly yield of the SES for the total area can be calculated (3) and depends on several correction factors [7]:

- $\quad V_{\text {incl. }}$, relation between a fixed and variable tilt angle

- $V_{\text {temp }}$, depending on the voltage loss of the solar panel

- $V_{\text {ref }}$, depending on the reflection coefficient of the solar panel (0,97 -3\% AAS-)

- $\mathrm{G}$, relation between the radiation measured on a horizontal surface and the radiation under standard test conditions $E=1000 \mathrm{~W} / \mathrm{m}^{2}$

$$
E_{P V}=P_{P V} \cdot G \cdot V_{\text {incl }} \cdot V_{\text {temp }} \cdot V_{\text {ref }} \quad[k W h]
$$

The yearly yield for the area North - West is estimated at 3,017GWh.

At the airport emergency power (NSA) is used for peak shaving. By peak shaving, the costs of the bought electricity can be reduced. Decentralized co-generators (CHP) are part of the electricity grid of AAS, but are not always used for peak shaving. 
The emergency power is switched on automatically when the maximal limit of the active power in the main station has been reached. The switching part is done by the Power Energy Control System (PECS) and measured by P', see figure 10 .

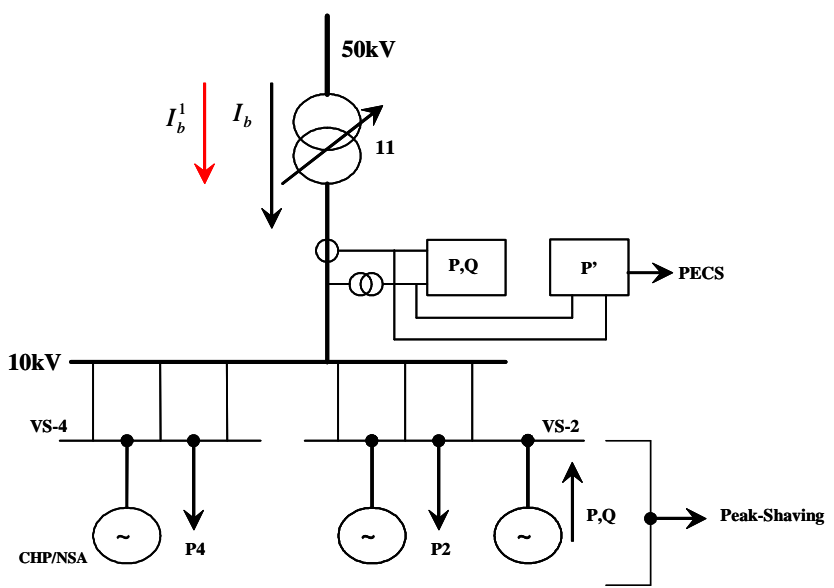

Figure 10 Peak shaving lay-out main station Schiphol

When a maximum limit of the total imported power has been reached the decentralized power will be switched or controlled by the PECS. The amount of decentralized power is given by $I^{\prime \prime}=I_{b}-I_{b}^{\prime}$ where $I_{b}=$ nominal current and $I_{b}^{\prime}=$ compensated current. Because of legal regulations for emissions, it is not wise to use emergency power for peak shaving purposes. The SES can be used for peak shaving and is comparable with a co-generator, see figure 11 .

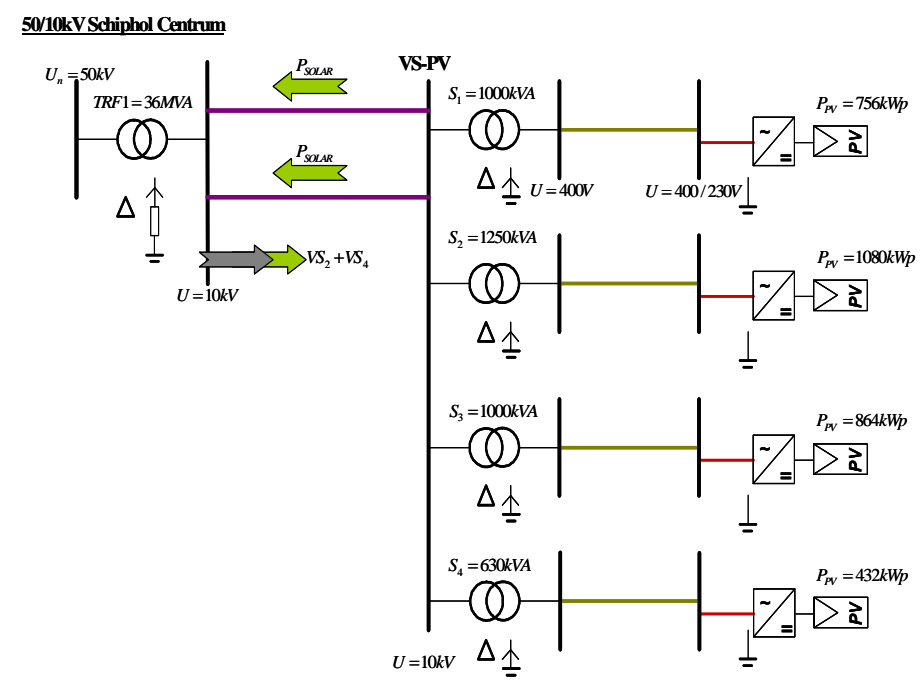

Figure 11 Solar plant part of load management

The generated power will directly be transported to the $50 / 10 \mathrm{kV}$ main station and coupled to the load of AAS. In this way, the co-generators and the solar plant are part of the load management. In this way the SES gives a positive contribution not only for the renewable part but also for the amount of the bought (imported) active power.

\section{Power Quality (PQ) and safety}

Power Quality describes the quality of the power exchanged at the point of connection and depends on the quality of the voltage and current. The distortion of the voltage level may not differ much from the ideal sinusoidal waveform, fundamental harmonic $50 \mathrm{~Hz}$ [8]. Due to the power system impedance, any current or voltage harmonic will result in generation and propagation of voltage and currents harmonics and affects the entire power system. The classifications for PQ are not identical for each issue and they are categorized in different guidelines e.g. IEC61000-2-5.

The effect of disturbances and behavior of appliances on PQ cannot be solved completely but it should be decreased to normal proportions. The quality of the main voltage depends on the electromagnetic compatibility (EMC) which depends on the electromagnetic interference (EMI). The EMI is defined as a disturbance signal that can lead to unacceptable working proportions. EMC is defined as the ability of the equipment or system to work in a proper way in the electromagnetic environment [9], see figure 12.

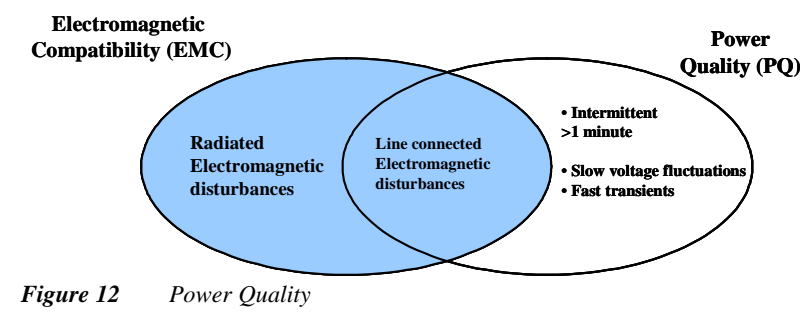

It is important to measure the amount of distortion and according to the results different actions can be taken to minimize the problem. With a SES harmonics will also be introduced into the system because the inverter causes harmonic distortion and the problem must be minimized by using an active or passive filter. It is also important to pay attention to the engineering of the grounding system for the installed equipment. The SES will be engineered as a TN-S system, see figure 13, where the ground is separate from the neutral conductor.

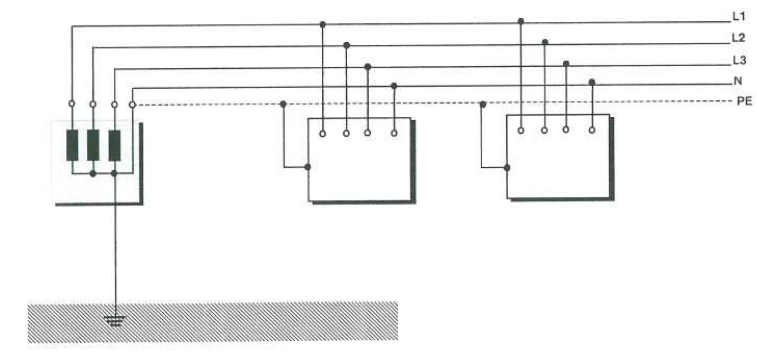

Figure 13 Grounding system $T N-S$

The ground system must lead to relative low impedance because when high currents occur with a specific frequency, dangerous voltages must be avoided. The current level of the neutral conductor must be very small. For the SES, the so-called stray capacitance is very important. Due to the structure of the solar module several parasitic capacitances occur, see figure 14. 
Depending of the generator setup and the used modules the capacitances are between $0,1 \ldots 100 \mathrm{nF} \ldots 1 \mu \mathrm{F}$.

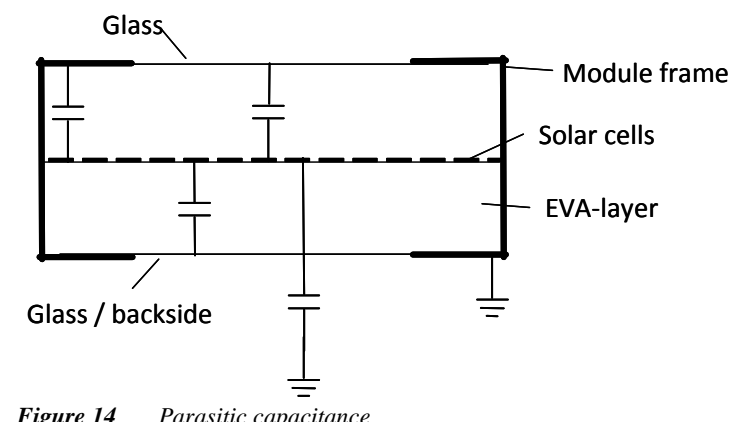

Attention also needs to be paid towards the cable system of the SES. The harmonics will lead to cable losses because of the magnetic field. This effect can be minimized by choosing a larger conductor cross-section. It has also to be noticed that the temperature of the insulation material, the positioning of the conductor itself and other conductors are important. For the SES a larger conductor cross section is chosen.

\section{Conclusion}

A solar energy system (SES) has been developed for Amsterdam Airport Schiphol (AAS). The design of the SES makes it possible to use it also for other Airports but the local constraints will have to be taken into account. Under no circumstances, the air flight safety should be affected negatively. The radiation reflection according to the visibility of the solar panels can be neglected and only depends on the distance, the intensity of the reflections and the reflection coefficient of the solar glass. The solar panels can be orientated on ground level and there are enough surfaces available - in line with the several guidelines for the communication, radar and surveillance equipment as well as for the building restricted areas. The solar panels will be positioned with a tilt angle of $\gamma_{P}=31^{\circ}$ and orientated in the South direction $\alpha_{P}=180^{\circ}$. The Power Quality effect needs to be evaluated and measured after the implementation of the SES and according to these measurements the problem can be minimized by e.g. filtering techniques.

\section{Acknowledgement}

The author would also like to thank a few people for their support during this research project and for their helpful suggestions: Sébastien Baumlin and Heiko Stieber.

\section{References}

[1] Schiphol Group, Climate plan Amsterdam Airport Schiphol, 2007

[2] International Civil Aviation Organization (ICAO) Aerodromes ANNEX14, third edition July 1999 Aeronautical Telecommunications ANNEX10, fifth edition July 1996

[3] Procesbeschrijving VOBA, MS/PR/LO/47842 Versie 1.0, februari 2008

[4] www.tudelft.nl /Information about Photometric quantities

[5] V. Quaschning, Understanding renewable energy systems, Eartscan 2005

[6] SMA Solar technology AG, Sunny mini Central 11000TL Manual

[7] J.M.A. Myrzik, course on distributed Generation 5N510 TU Eindhoven

[8] J.F.G. Cobben, J.N. Luttjehuizen , Power Quality, Over spanning - stroom en hun interactie, SDu 2009

[9] E.F. Fuchs. M.A.S. Masoum, Power Quality in Power systems and Electrical machines, Academic press 2008 